\title{
Multiple solutions of a sublinear Schrödinger equation $^{1}$
}

\author{
Alexandru KRISTÁLY \\ University of Babeş-Bolyai \\ Department of Economics \\ 400147 Cluj-Napoca, Romania \\ e-mail: alexandrukristaly@yahoo.com
}

Abstract. In this paper we study the Schrödinger equation of the form

$\left(\mathrm{P}_{\lambda}\right)$

$$
-\triangle u+a(x) u=\lambda b(x) f(u), x \in \mathbb{R}^{N}, u \in H^{1}\left(\mathbb{R}^{N}\right),
$$

where $\lambda>0$ is a parameter, $a$ and $b$ are positive potentials, while the nonlinear term $f: \mathbb{R} \rightarrow \mathbb{R}$ is sublinear at infinity. Two cases will be considered: (i) $f$ is superlinear at the origin; (ii) $f$ does not satisfy any asymptotical property at the origin. In both situations, the existence of certain multiple weak solutions of $\left(\mathrm{P}_{\lambda}\right)$ are established for some $\lambda>0$.

2000 Mathematics Subject Classification: 35J60, 35J65.

Key words: Nonlinear Schrödinger equations; Sublinearity at infinity; Multiple solutions.

\section{Introduction and main results}

In this paper we consider the Schrödinger equation of the form

$$
-\triangle u+a(x) u=\lambda b(x) f(u), x \in \mathbb{R}^{N}, u \in H^{1}\left(\mathbb{R}^{N}\right),
$$

which depends on the parameter $\lambda>0$. Throughout the paper we assume that $\left(f_{0}\right) f \in \mathcal{C}(\mathbb{R}, \mathbb{R})$, and there exist $c>0$ and $\left.q \in\right] 0,1[$ such that

$$
|f(s)| \leq c|s|^{q} \text { for each } s \in \mathbb{R} \text {. }
$$

\footnotetext{
${ }^{1}$ This work was supported by the Istituto Nazionale di Alta Matematica, when the author visited the Department of Mathematics of the University of Catania, Italy, in June-July, 2005.
} 
Our interest in $\left(\mathrm{P}_{\lambda}\right)$, under hypothesis $\left(f_{0}\right)$, is motivated mainly by several papers where the nonlinearity $f$ is assumed to be superlinear at infinity, i.e., there exist some numbers $C>0, s_{0}>0$ and $p>1$ such that

$$
|f(s)| \geq C|s|^{p} \text { for each }|s| \geq s_{0} \text {. }
$$

In particular, studying closely related problems to $\left(\mathrm{P}_{\lambda}\right)$, many authors use the Ambrosetti-Rabinowitz type condition (see [2], [4], [5]) or some of its variant (see $[1],[8],[7])$ :

$(A R)$ There is $\eta>2$ such that

$$
0<\eta F(s) \leq f(s) s \text { for each } s \in \mathbb{R} \backslash\{0\},
$$

where $F(s)=\int_{0}^{s} f(t) d t$.

And as we know, condition $(A R)$ implies (1) with $p=\eta-1$, excluding clearly $\left(f_{0}\right)$.

The potential $a: \mathbb{R}^{N} \rightarrow \mathbb{R}$ also has an important role concerning the existence and asymptotic behaviour of solutions of $\left(\mathrm{P}_{\lambda}\right)$. When $a(x)=$ const. $>0$, or $a$ is radially symmetric, it is natural to look for radially symmetric solutions of $\left(\mathrm{P}_{\lambda}\right)$, see e.g., [5], [10], [11], [17], [19]. Apart from [11], in the aforementioned papers the nonlinearity $f$ fulfills $(A R)$. Motivated by the work of Rabinowitz [12] (where $a \in \mathcal{C}\left(\mathbb{R}^{N}, \mathbb{R}\right)$, $\inf _{\mathbb{R}^{N}} a>0$, and $a(x) \rightarrow+\infty$ as $\left.|x| \rightarrow+\infty\right)$, Bartsch and Wang [4] considered more general potentials:

$(B W) a \in \mathcal{C}\left(\mathbb{R}^{N}, \mathbb{R}\right)$ satisfies $\inf _{\mathbb{R}^{N}} a>0$, and for any $M>0$

$$
\mu\left(\left\{x \in \mathbb{R}^{N}: a(x) \leq M\right\}\right)<+\infty,
$$

where $\mu$ denotes the Lebesgue measure in $\mathbb{R}^{N}$.

Under $(B W)$, Bartsch and Wang [4] proved the existence of infinitely many solutions of $\left(\mathrm{P}_{\lambda}\right)$ (with $b(x)=$ const. $>0$ and any fixed $\lambda>0$ ) when $f: \mathbb{R} \rightarrow \mathbb{R}$ is subcritical, odd and verifies $(A R)$. Furtado, Maia and Silva [7] studied $\left(\mathrm{P}_{\lambda}\right)$ in the case when $F: \mathbb{R} \rightarrow \mathbb{R}$ (defined in $(A R)$ ) has some sort of resonance with a local nonquadraticity condition at infinity, while the potential $a$ verifies $(B W)$. Gazzola and Rădulescu [8] studied $\left(\mathrm{P}_{\lambda}\right)$ when $a$ verifies $(B W), f$ is not necessarily continuous and satisfies an appropriate non-smooth $(A R)$ condition.

In this paper we will use a weaker condition than $(B W)$, see Bartsch, Pankov and Wang [3]. Namely, we assume

$\left(a_{0}\right) a \in L_{\text {loc }}^{\infty}\left(\mathbb{R}^{N}\right), \operatorname{essinf}_{\mathbb{R}^{N}} a>0$, and

for any $M>0$ and any $r>0$ there holds:

$$
\mu(\{x \in B(y, r): a(x) \leq M\}) \rightarrow 0 \text { as }|y| \rightarrow+\infty,
$$

where $B(y, r)$ denotes the open ball in $\mathbb{R}^{N}$ with center $y$ and radius $r>0$. 
Requiring $\left(a_{0}\right)$, Bartsch, Liu and Weth [2] proved recently the existence of three solutions of $\left(\mathrm{P}_{\lambda}\right)$ (a positive, a negative, and a sign changing solution), e.g., if $b(x)=$ const. $>0, \lambda>0$ is arbitrarily fixed, $f$ is subcritical and verifies $(A R)$. Due to $\left(a_{0}\right)$, the Hilbert space

$$
E=\left\{u \in H^{1}\left(\mathbb{R}^{N}\right): \int_{\mathbb{R}^{N}} a(x) u^{2}<+\infty\right\}
$$

endowed with the inner product

$$
(u, v)_{E}=\int_{\mathbb{R}^{N}}(\nabla u \nabla v+a(x) u v) \text { for each } u, v \in E
$$

(and with the induced norm $\|\cdot\|_{E}$ ) can be continuously embedded into $L^{l}\left(\mathbb{R}^{N}\right)$ whenever $2 \leq l \leq 2^{*}$, and the embedding $E \hookrightarrow L^{l}\left(\mathbb{R}^{N}\right)$ is compact when $2 \leq$ $l<2^{*}$, cf. Bartsch, Pankov and Wang [3]. Here, $2^{*}$ denotes the critical Sobolev exponent, i.e., $2^{*}=2 N /(N-2)$ for $N \geq 3$ and $2^{*}=+\infty$ for $N=1,2$.

Since $f(0)=0$ (cf. $\left.\left(f_{0}\right)\right)$, the element 0 is a solution of $\left(\mathrm{P}_{\lambda}\right)$ for every $\lambda>0$. In order to guarantee nonzero solutions for $\left(\mathrm{P}_{\lambda}\right)$ we require the following assumptions:

$\left(b_{0}\right) b \in L^{1}\left(\mathbb{R}^{N}\right) \cap L^{\infty}\left(\mathbb{R}^{N}\right), b \geq 0$, and

$$
\sup _{R>0} \operatorname{essinf} f_{|x| \leq R} b(x)>0 .
$$

$\left(f_{1}\right) f(s)=o(|s|)$ as $s \rightarrow 0$.

$\left(f_{2}\right) \sup _{s \in \mathbb{R}} F(s)>0$.

Theorem 1.1 Suppose $\left(a_{0}\right),\left(b_{0}\right)$ and $\left(f_{0}\right)-\left(f_{2}\right)$ hold. Then there exist an open interval $\Lambda \subseteq(0, \infty)$ and a number $\nu>0$ such that for every $\lambda \in \Lambda$ problem $\left(\mathrm{P}_{\lambda}\right)$ has at least two distinct nonzero weak solutions $u_{\lambda}^{i}(i \in\{1,2\})$ such that $\left\|u_{\lambda}^{i}\right\|_{E} \leq \nu$ for every $\lambda \in \Lambda$ and $i \in\{1,2\}$. In addition, if $f(s)=0$ for each $s \in]-\infty, 0]$, the solutions $u_{\lambda}^{i}$ are non-negative.

Not only in Theorem 1.1 but also in the aforementioned papers ([2], [4], [5], $[8],[16],[17],[19])$, the superlinearity of $f$ at the origin (i.e. hypothesis $\left(f_{1}\right)$ ) is an indispensable fact. The aim of the next result is to handle the situation when we drop $\left(f_{1}\right)$. As it is expected, this step will be penalized: instead of standard weak solutions of $\left(\mathrm{P}_{\lambda}\right)$ we will be able only to obtain multiple solutions for a closely related (perturbed) problem to $\left(\mathrm{P}_{\lambda}\right)$. To state this result precisely, let us define the functional $\mathcal{F}: E \rightarrow \mathbb{R}$ for each $u \in E$ by

$$
\mathcal{F}(u)=\int_{\mathbb{R}^{N}} b(x) F(u(x)) d x .
$$


Theorem 1.2 Suppose $\left(a_{0}\right),\left(b_{0}\right)$ and $\left(f_{0}\right)$ hold, and in addition $f: \mathbb{R} \rightarrow \mathbb{R}$ is a nonzero, non-decreasing function. Then for each number $\zeta>0$ there exist a number $\lambda>0$ and $w \in \mathcal{F}^{-1}\left(\left[0, \zeta[) \cap C_{0}^{\infty}\left(\mathbb{R}^{N}\right)\right.\right.$ such that the problem

$$
-\triangle u+a(x) u=\lambda b(x) f(u+w), x \in \mathbb{R}^{N}
$$

has at least three distinct weak solutions.

We emphasize that the conclusions of Theorems 1.1 and 1.2 do not hold generally for every $\lambda>0$. For detailed comments, see Section 4 . In the next two sections we will prove Theorems 1.1 and 1.2, respectively, applying two recent critical point results of Ricceri $[13,15]$.

\section{Proof of Theorem 1.1}

A standard argument, which is based on the facts that $b \in L^{1}\left(\mathbb{R}^{N}\right) \cap L^{\infty}\left(\mathbb{R}^{N}\right), f$ satisfies $\left(f_{0}\right)$, and the embedding $E \hookrightarrow L^{p}\left(\mathbb{R}^{N}\right)$ is continuous $\left(2 \leq p \leq 2^{*}\right)$, shows that the functional $\mathcal{F}: E \rightarrow \mathbb{R}$, introduced in $(3)$, is well defined, is of class $\mathcal{C}^{1}$, and satisfies

$$
\mathcal{F}^{\prime}(u)(v)=\int_{\mathbb{R}^{N}} b(x) f(u(x)) v(x) \text { for each } u, v \in E .
$$

Moreover, since $E \hookrightarrow L^{p}\left(\mathbb{R}^{N}\right)$ is compact $\left(2 \leq p<2^{*}\right)$ one can show that $\mathcal{F}^{\prime}$ is a compact operator. (For a similar argument, see Gonçalves and Miyagaki [9].) In the sequel, we denote by $\kappa_{l}>0$ the Sobolev embedding constant for $E \hookrightarrow L^{l}\left(\mathbb{R}^{N}\right), l \in\left[2,2^{*}\right.$, while $\|\cdot\|_{L^{l}}$ denotes the usual norm of $L^{l}\left(\mathbb{R}^{N}\right), 1 \leq l \leq \infty$.

Define now the functional $\mathcal{H}: E \times] 0,+\infty[\rightarrow \mathbb{R}$ by

$$
\left.\mathcal{H}(u, \lambda)=\frac{1}{2}\|u\|_{E}^{2}-\lambda \mathcal{F}(u) \text { for each }(u, \lambda) \in E \times\right] 0,+\infty[.
$$

The weak solutions of the problem $\left(\mathrm{P}_{\lambda}\right)$ are precisely the critical points of $\mathcal{H}(\cdot, \lambda)$.

Lemma 2.1 $\lim _{\rho \rightarrow 0^{+}} \frac{\sup \left\{\mathcal{F}(u):\|u\|_{E}<\sqrt{2 \rho}\right\}}{\rho}=0$.

Proof. Fix $\varepsilon>0$ arbitrarily and a number $2<p<2^{*}$. Due to $\left(f_{0}\right)$ and $\left(f_{1}\right)$, there exists $c(\varepsilon)>0$ such that

$$
|f(s)|<\varepsilon\left(4\|b\|_{L^{\infty}} \kappa_{2}^{2}\right)^{-1}|s|+c(\varepsilon)|s|^{p-1} \text { for each } s \in \mathbb{R} .
$$

For $\rho>0$ define the set $A_{\rho}=\left\{u \in E:\|u\|_{E}<\sqrt{2 \rho}\right\}$. Due to (5), for every $u \in A_{\rho}$ we have

$$
\begin{aligned}
\mathcal{F}(u) & \leq \int_{\mathbb{R}^{N}} b(x)|F(u(x))| d x \leq\|b\|_{L^{\infty}}\left(\varepsilon\left(4\|b\|_{L^{\infty}} \kappa_{2}^{2}\right)^{-1}\|u\|_{L^{2}}^{2}+c(\varepsilon)\|u\|_{L^{p}}^{p}\right) \\
& \leq \frac{\varepsilon}{2} \rho+\|b\|_{L^{\infty}} c(\varepsilon) \kappa_{p}^{p}(2 \rho)^{p / 2} .
\end{aligned}
$$


Thus there exists $\rho(\varepsilon)>0$ such that for every $0<\rho<\rho(\varepsilon)$

$$
0 \leq \frac{\sup _{u \in A_{\rho}} \mathcal{F}(u)}{\rho} \leq \frac{\varepsilon}{2}+c^{\prime}(\varepsilon) \rho^{\frac{p-2}{2}}<\varepsilon,
$$

which completes the proof.

Lemma 2.2 For any $\lambda>0$, the functional $\mathcal{H}(\cdot, \lambda)$ is sequentially weakly lower semicontinuous and satisfies the Palais-Smale condition.

Proof. Since $\mathcal{F}^{\prime}$ is a compact operator, the functional $\mathcal{F}$ is sequentially weakly continuous, see [20, Corollary 41.9]. Since the function $E \ni u \mapsto\|u\|_{E}^{2}$ is sequentially weakly lower semicontinuous, the first statement holds.

Fix $\lambda>0$. Since $\mathcal{H}^{\prime}(\cdot, \lambda)(u)=u-\lambda \mathcal{F}^{\prime}(u), u \in E$, in view of $[20$, Example $38.25]$ it is enough to show the coercivity of $\mathcal{H}(\cdot, \lambda)$. By $\left(f_{0}\right)$ and $\left(b_{0}\right)$ one has

$$
\mathcal{H}(u, \lambda)=\frac{1}{2}\|u\|_{E}^{2}-\lambda \mathcal{F}(u) \geq \frac{1}{2}\|u\|_{E}^{2}-\lambda c \kappa_{2}^{q+1}\|b\|_{L^{2 /(1-q)}}\|u\|_{E}^{q+1} .
$$

Since $q<1$, then $\mathcal{H}(u, \lambda) \rightarrow+\infty$ as $\|u\|_{E} \rightarrow+\infty$, as claimed.

The main ingredient in the proof of Theorem 1.1 is a Ricceri-type critical point theorem, see $[14,15]$. Here, we recall a refinement of this result, established by Bonanno [6].

Theorem 2.1 (see [6, Theorem 2.1]) Let $X$ be a separable and reflexive real Banach space, and let $\Phi, J: X \rightarrow \mathbb{R}$ be two continuously Gâteaux differentiable functionals. Assume that there exists $x_{0} \in X$ such that $\Phi\left(x_{0}\right)=J\left(x_{0}\right)=0$ and $\Phi(x) \geq 0$ for every $x \in X$ and that there exists $x_{1} \in X, \rho>0$ such that

(i) $\rho<\Phi\left(x_{1}\right)$ and $\sup _{\Phi(x)<\rho} J(x)<\rho \frac{J\left(x_{1}\right)}{\Phi\left(x_{1}\right)}$.

Further, put

$$
\bar{a}=\frac{\zeta \rho}{\rho \frac{J\left(x_{1}\right)}{\Phi\left(x_{1}\right)}-\sup _{\Phi(x)<\rho} J(x)},
$$

with $\zeta>1$, assume that the functional $\Phi-\lambda J$ is sequentially weakly lower semicontinuous, satisfies the Palais-Smale condition and

(ii) $\lim _{\|x\| \rightarrow+\infty}(\Phi(x)-\lambda J(x))=+\infty$,

for every $\lambda \in[0, \bar{a}]$.

Then there is an open interval $\Lambda \subset[0, \bar{a}]$ and a number $\nu>0$ such that for each $\lambda \in \Lambda$, the equation $\Phi^{\prime}(x)-\lambda J^{\prime}(x)=0$ admits at least three distinct solutions in $X$ having norm less than $\nu$.

Proof of Theorem 1.1. Apply Theorem 2.1 with $X=E, \Phi=\frac{1}{2}\|\cdot\|_{E}^{2}$ $J=\mathcal{F}$. Due to $\left(b_{0}\right)$ and $\left(f_{2}\right)$, there exist $R_{0}>0$ and $s_{0} \in \mathbb{R}$ such that $b_{R_{0}}=$ 
$\operatorname{essinf}_{|x| \leq R_{0}} b(x)>0$ and $F\left(s_{0}\right)>0$, respectively. Choose further a number $0<$ $\varepsilon<1$ such that

$$
b_{R_{0}} F\left(s_{0}\right) \varepsilon^{N}-\|b\|_{L^{\infty}} \max _{\left[-\left|s_{0}\right|,\left|s_{0}\right|\right]} F\left(1-\varepsilon^{N}\right)>0 .
$$

Moreover, let $u_{\varepsilon} \in E$ such that $u_{\varepsilon}(x)=s_{0}$ for any $x \in B\left(0, \varepsilon R_{0}\right), u_{\varepsilon}(x)=0$ for any $x \in \mathbb{R}^{N} \backslash B\left(0, R_{0}\right)$, and $\left\|u_{\varepsilon}\right\|_{L^{\infty}} \leq\left|s_{0}\right|$. Denoting by $\omega_{N}$ the volume of the unit ball in $\mathbb{R}^{N}$, by means of (6) one has

$$
\mathcal{F}\left(u_{\varepsilon}\right) \geq b_{R_{0}} F\left(s_{0}\right) \varepsilon^{N} R_{0}^{N} \omega_{N}-\|b\|_{L^{\infty}} \max _{\left[-\left|s_{0}\right|,\left|s_{0}\right|\right]} F\left(1-\varepsilon^{N}\right) R_{0}^{N} \omega_{N}>0 .
$$

Due to Lemma 2.1, one can fix a small number $\rho=\rho(\varepsilon)>0$ such that $\sqrt{2 \rho}<$ $\left\|u_{\varepsilon}\right\|_{E}$ and

$$
\frac{\sup \left\{\mathcal{F}(u):\|u\|_{E}<\sqrt{2 \rho}\right\}}{\rho}<\frac{2 \mathcal{F}\left(u_{\varepsilon}\right)}{\left\|u_{\varepsilon}\right\|_{E}^{2}} .
$$

Therefore, choosing $x_{0}=0, x_{1}=u_{\varepsilon}, \zeta=1+\varepsilon$, and taking into account Lemma 2.2, the hypotheses of Theorem 2.1 are verified with

$$
\bar{a}=\frac{1+\varepsilon}{2 \mathcal{F}\left(u_{\varepsilon}\right)\left\|u_{\varepsilon}\right\|_{E}^{-2}-\sup \left\{\mathcal{F}(u):\|u\|_{E}<\sqrt{2 \rho}\right\} \rho^{-1}} .
$$

Then there is an open interval $\Lambda \subset[0, \bar{a}]$ and a number $\nu>0$ such that for any $\lambda \in \Lambda$, the functional $\mathcal{H}(\cdot, \lambda)$ admits at least three distinct critical points $u_{\lambda}^{i} \in E$ $(i \in\{1,2,3\})$, having norm less than $\nu$, concluding the first part of Theorem 1.1. When $f \equiv 0$ on the set $]-\infty, 0]$, a standard argument shows that the negative part of a solution $u_{\lambda}^{i}$ should be zero, as claimed.

\section{Proof of Theorem 1.2}

Lemma 3.1 $\inf _{E} \mathcal{F}=0 ; \sup _{E} \mathcal{F}=+\infty$.

Proof. Since $f(0)=0$ (see $\left(f_{0}\right)$ ), and $f$ is non-decreasing, it is easy to prove that the function $F(s)=\int_{0}^{s} f(t) d t$ is non-negative for any $s \in \mathbb{R}$. Since $\mathcal{F}(0)=0$, and the potential $b$ is non-negative, see $\left(b_{0}\right)$, the first assertion of the lemma holds. Furthermore, since $f$ is nonzero and non-decreasing, then $\sup _{\mathbb{R}} F=+\infty$. Let $\left\{a_{n}\right\} \subset \mathbb{R}$ be a sequence such that $F\left(a_{n}\right) \rightarrow+\infty$ as $n \rightarrow+\infty$. By $\left(b_{0}\right)$, one can fix $R_{0}>0$ such that $b_{R_{0}}=\operatorname{essinf}_{|x| \leq R_{0}} b(x)>0$. Let $\left\{u_{n}\right\} \subset E$ be such that $u_{n}(x)=a_{n}$ for any $x \in B\left(0, R_{0}\right)$. Since $b$ and $F$ are non-negative functions, one has

$$
\sup _{E} \mathcal{F} \geq \mathcal{F}\left(u_{n}\right) \geq b_{R_{0}} F\left(a_{n}\right) R_{0}^{N} \omega_{N} \rightarrow+\infty
$$

as $n \rightarrow+\infty$, as claimed. 
Lemma 3.2 Let $\zeta>0$ and $\gamma \in \mathbb{R}$ be two fixed numbers. Then there exist $u=$ $u(\zeta, \gamma) \in E$ and $r=r(\zeta, \gamma)>0$ such that $\mathcal{F}(u)=\zeta$, and $u(x)=\gamma$ for any $x \in B(0, r)$.

Proof. Fix $\bar{\zeta} \in] \zeta,+\infty\left[\right.$, and $R_{0}>0$ such that $b_{R_{0}}=\operatorname{essinf}_{|x| \leq R_{0}} b(x)>0\left(\operatorname{cf} .\left(b_{0}\right)\right)$. Fix $r>0$ so small that

$$
\begin{gathered}
2 r<R_{0} ; \\
\|b\|_{L^{\infty}} \max _{[-|\gamma|,|\gamma|]} F \omega_{N}(2 r)^{N}<\zeta ; \\
\bar{\zeta}\left(1-\left(2 r / R_{0}\right)^{N}\right)>\zeta .
\end{gathered}
$$

Let $u_{0} \in E$ be such that $u_{0}(x)=\gamma$ for any $x \in B(0, r), u_{0}(x)=0$ for any $x \notin B(0,2 r)$, and $\left\|u_{0}\right\|_{L^{\infty}} \leq|\gamma|$. Then, due to (8) one has

$$
\begin{aligned}
\mathcal{F}\left(u_{0}\right) & =\int_{\mathbb{R}^{N}} b(x) F\left(u_{0}(x)\right) d x \\
& \leq\|b\|_{L^{\infty}} F(\gamma) \omega_{N} r^{N}+\|b\|_{L^{\infty}} \max _{[-|\gamma|,|\gamma|]} F \omega_{N}\left(2^{N}-1\right) r^{N} \\
& <\zeta .
\end{aligned}
$$

On the other hand, since $\inf _{\mathbb{R}} F=0$ and $\sup _{\mathbb{R}} F=+\infty$, there exists $\bar{\xi} \in \mathbb{R}$ such that

$$
F(\bar{\xi})=\bar{\zeta}\left(b_{R_{0}} \omega_{N} R_{0}^{N}\right)^{-1}
$$

According to (7), we may define $u_{1} \in E$ such that $u_{1}(x)=\gamma$ for any $x \in B(0, r)$, and $u_{1}(x)=\bar{\xi}$ for any $x \in B\left(0, R_{0}\right) \backslash B(0,2 r)$. Since the functions $b$ and $F$ are non-negative, by (9) and (10) we have

$$
\mathcal{F}\left(u_{1}\right) \geq F(\bar{\xi}) \operatorname{essinf}_{2 r \leq|x| \leq R_{0}} b(x) \omega_{N}\left(R_{0}^{N}-(2 r)^{N}\right)>\zeta .
$$

Define the set

$$
S_{r}^{\gamma}=\{u \in E: u(x)=\gamma \text { for each } x \in B(0, r)\}
$$

Taking into account the above constructions, we have two elements $u_{0}, u_{1} \in S_{r}^{\gamma}$ such that $\mathcal{F}\left(u_{0}\right)<\zeta<\mathcal{F}\left(u_{1}\right)$. Since the function $E \ni u \mapsto \mathcal{F}(u)$ is continuous and the set $S_{r}^{\gamma}$ is connected (because it is convex), then there exists $u \in S_{r}^{\gamma}$ such that $\mathcal{F}(u)=\zeta$.

Lemma 3.3 Let $\zeta>0$ be a fixed number. Then the set $\mathcal{F}^{-1}([\zeta,+\infty[)$ is not convex. 
Proof. Since $f$ is non-decreasing, then $\mathcal{F}^{\prime}$ is monotone, cf. (4) and $\left(b_{0}\right)$. Therefore, $\mathcal{F}$ is a convex function and the level set $\left.\left.\mathcal{F}^{-1}(]-\infty, \zeta\right]\right)$ is convex. If we assume that $\mathcal{F}^{-1}\left(\left[\zeta,+\infty[)\right.\right.$ is also convex, then $\left.\left.\mathcal{F}^{-1}(\zeta)=\mathcal{F}^{-1}(]-\infty, \zeta\right]\right) \cap \mathcal{F}^{-1}([\zeta,+\infty[)$ will be convex as well. Thus, in order to get the conclusion of the lemma, it is enough to prove that $\mathcal{F}^{-1}(\zeta)$ is not convex.

To this end, recall that $f(0)=0$ and $f$ is a nonzero function, i.e., there exists $\gamma \in \mathbb{R}$ such that $f(\gamma) \neq f(0)=0$. By Lemma 3.2, there exist $u_{0}, u_{1} \in E$ and $r>0$ such that $u_{0}(x)=0$ and $u_{1}(x)=\gamma$ for any $x \in B(0, r)$, and $u_{0}, u_{1} \in \mathcal{F}^{-1}(\zeta)$. Arguing by contradiction, suppose that

$$
\mathcal{F}\left(t u_{0}+(1-t) u_{1}\right)=\zeta \text { for each } t \in[0,1] .
$$

After a differentiation in (11) in rapport of $t$ and by using (4) one has

$$
\int_{\mathbb{R}^{N}} b(x) f\left(t u_{0}(x)+(1-t) u_{1}(x)\right)\left(u_{0}(x)-u_{1}(x)\right) d x=0
$$

for any $t \in[0,1]$. Choosing in particular $t=0$ and $t=1$ in the above relation, one has

$$
\int_{\mathbb{R}^{N}} b(x)\left[f\left(u_{0}(x)\right)-f\left(u_{1}(x)\right)\right]\left(u_{0}(x)-u_{1}(x)\right) d x=0 .
$$

Since the potential $b$ is non-negative and $f$ is non-decreasing, we obtain

$$
b(x)\left[f\left(u_{0}(x)\right)-f\left(u_{1}(x)\right)\right]\left(u_{0}(x)-u_{1}(x)\right)=0 \text { for a.e. } x \in \mathbb{R}^{N} .
$$

On the other hand, hypothesis $\left(b_{0}\right)$ asserts the existence of a number $R_{0}>0$ such that $b_{R_{0}}=\operatorname{essinf}|x| \leq R_{0} b(x)>0$. Now, applying the last relation on the ball $B\left(0, \min \left\{R_{0}, r\right\}\right)$ and exploring the choice of $u_{0}$ and $u_{1}$, respectively, we are led to $f(\gamma) \gamma=0$, which contradicts the choice of the number $\gamma$. Thus, (11) is false, i.e., the set $\mathcal{F}^{-1}(\zeta)$ is not convex.

Now, we are in the position to prove Theorem 1.2. To do this, we recall another recent critical point theorem of Ricceri which is derived by an ingenious application of a recent result of Tsar'kov [18], and it was applied to solve a two point boundary value problem for ordinary differential equations, see [13].

Theorem 3.1 (see [13, Theorem 2]) Let $X$ be a real Hilbert space and $J: X \rightarrow \mathbb{R}$ a continuous Gâteaux differentiable, nonconstant functional, with compact derivative, such that

$$
\limsup _{\|x\| \rightarrow+\infty} \frac{J(x)}{\|x\|^{2}} \leq 0 .
$$

Then, for each $r \in] \inf _{X} J, \sup _{X} J\left[\right.$ for which the set $J^{-1}([r,+\infty[)$ is not convex and for every set $S \subseteq X$ dense in $X$, there exist $x_{0} \in J^{-1}(]-\infty, r[) \cap S$ and $\lambda>0$ such that the equation

$$
x=\lambda J^{\prime}(x)+x_{0}
$$

has at least three distinct solutions. 
Proof of Theorem 1.2. We apply Theorem 3.1 by choosing $X=E$, $J=\mathcal{F}$, and $S=C_{0}^{\infty}\left(\mathbb{R}^{N}\right)$. As in the previous section, we have that $\mathcal{F}$ is of class $\mathcal{C}^{1}$, and $\mathcal{F}^{\prime}$ is compact. Indeed, here we used only $\left(f_{0}\right)$ and the fact that $b \in L^{1}\left(\mathbb{R}^{N}\right) \cap L^{\infty}\left(\mathbb{R}^{N}\right)$. Lemma 3.2 implies in particular that $\mathcal{F}$ is not a constant functional. By using $\left(f_{0}\right)$, one has

$$
\mathcal{F}(u)=\int_{\mathbb{R}^{N}} b(x) F(u(x)) d x \leq c \kappa_{2}^{q+1}\|b\|_{L^{2 /(1-q)}}\|u\|_{E}^{q+1} .
$$

Thus, the inequality (12) is clearly verified, since $q<1$. Taking into account Lemmas 3.1 and 3.3 , for every $\zeta \in] 0,+\infty\left[\right.$ there exist $w \in \mathcal{F}^{-1}(]-\infty, \zeta[) \cap$ $C_{0}^{\infty}\left(\mathbb{R}^{N}\right)=\mathcal{F}^{-1}\left(\left[0, \zeta[) \cap C_{0}^{\infty}\left(\mathbb{R}^{N}\right)\right.\right.$ and $\lambda>0$ such that the equation

$$
v=\lambda \mathcal{F}^{\prime}(v)+w,
$$

has three distinct solutions, say $v_{i} \in E, i \in\{1,2,3\}$. Due to (13) and (4), the elements $v_{i}$ are weak solutions of the equation

$$
-\triangle v+a(x) v=\lambda b(x) f(v)-\triangle w+a(x) w, x \in \mathbb{R}^{N} .
$$

Therefore, the elements $u_{i}=v_{i}-w$ are weak solutions of $\left(\mathrm{P}_{\lambda}^{w}\right)$. This concludes the proof.

\section{Concluding remarks}

A.) In Theorems 1.1 and 1.2 , the inequality (2) in hypothesis $\left(b_{0}\right)$ cannot be dropped (see also [11] for a similar argument). If we omit (2), we can simply take $b \equiv 0$, obtaining only the trivial solution for $\left(\mathrm{P}_{\lambda}\right)$. Moreover, the non-negativity of the potential $b$ is exploited in the proof of Theorem 1.2.

B.) In spite of the fact that hypotheses of Theorems 1.1 and 1.2 are verified, the conclusions do not hold in general for every parameter $\lambda>0$, as we mentioned in the first section. In the sequel, we give two such examples.

B1. Let $f(s)=(\arctan (s))^{2}$. The function $f$ verifies clearly $\left(f_{0}\right)-\left(f_{2}\right)$. However, if $0<\lambda<\left(\pi\|b\|_{L^{\infty}} \kappa_{2}^{2}\right)^{-1}$, the problem $\left(\mathrm{P}_{\lambda}\right)$ has only the zero solution. Indeed, observe that the critical points of $\mathcal{H}(\cdot, \lambda)$ are precisely the fixed points of the operator $A_{\lambda}=\lambda \mathcal{F}^{\prime}$. Since $A_{\lambda}$ is a contraction for the abovespecified $\lambda^{\prime} \mathrm{s}, A_{\lambda}$ has a unique fixed point. Since $A_{\lambda}(0)=0$ (due to the fact that $f(0)=0)$, then 0 will be the unique solution of $\left(\mathrm{P}_{\lambda}\right)$.

B2. Let $f(s)=\arctan (s)$. The function $f$ verifies $\left(f_{0}\right)$ (but not $\left(f_{1}\right)$ ). Whenever $0<\lambda<\left(\|b\|_{L^{\infty}} \kappa_{2}^{2}\right)^{-1}$, the problem $\left(\mathrm{P}_{\lambda}^{w}\right)$ has a unique solution for every element $w \in C_{0}^{\infty}\left(\mathbb{R}^{N}\right)$. Indeed, for a fixed $w \in C_{0}^{\infty}\left(\mathbb{R}^{N}\right)$, the weak solutions of $\left(\mathrm{P}_{\lambda}^{w}\right)$ appear as $v-w$, where the elements $v$ are fixed points of the operator $A_{\lambda}^{w}(v)=\lambda \mathcal{F}^{\prime}(v)+w(\mathrm{cf}$. (13)), which is a contraction for each $\lambda \in] 0,\left(\|b\|_{L^{\infty}} \kappa_{2}^{2}\right)^{-1}\left[\right.$. Thus $A_{\lambda}^{w}$ has a unique fixed point. 


\section{Acknowledgement}

The author would like to thank Professor Biagio Ricceri for fruitful and stimulating discussions.

\section{References}

[1] P. BARTOLO, V. BENCI and D. FORTUNATO, Abstract critical point theorems and applications to some nonlinear problems with "strong" resonance at infinity, Nonlinear Anal. 7 (1983), 981-1012.

[2] T. BARTSCH, Z. LIU and T. WETH, Sign changing solutions of superlinear Schrödinger equations, Comm. Partial Differential Equations, 29(2004), $25-42$.

[3] T. BARTSCH, A. PANKOV and Z.-Q. WANG, Nonlinear Schrödinger equations with steep potential well, Comm. Contemp. Math. 4 (2001), 549-569.

[4] T. BARTSCH and Z.-Q. WANG, Existence and multiplicity results for some superlinear elliptic problems on $\mathbb{R}^{N}$, Comm. Partial Differential Equations, 20 (1995), 1725-1741.

[5] T. BARTSCH and M. WILLEM, Infinitely many non-radial solutions of an Euclidean scalar field equation, J. Func. Anal. 117 (1995), 447-460.

[6] G. BONANNO, Some remarks on a three critical points theorem, Nonlinear Analysis TMA, 54 (2003), 651-665.

[7] M. F. FURTADO, L. A. MAIA and E. A. B. SILVA, On a double resonant problem in $\mathbb{R}^{N}$, Differential Integral Equations, 15 (2002), 1335-1344.

[8] F. GAZZOLA and V. RĂDULESCU, A nonsmooth critical point theory approach to some nonlinear elliptic equations in $\mathbb{R}^{N}$, Differential Integral Equations, 13 (2000), 47-60.

[9] J. V. GONÇALVES and O. H. MIYAGAKI, Multiple positive solutions for semilinear elliptic equations in $\mathbb{R}^{N}$ involving subcritical exponents, Nonlinear Analysis TMA, 32 (1998), 41-51.

[10] A. KRISTÁLY, Infinitely many radial and non-radial solutions for a class of hemivariational inequalities, Rocky Mountain J. Math. 35(2005), 1173-1190.

[11] A. KRISTÁLY and CS. VARGA, On a class of quasilinear eigenvalue problems in $\mathbb{R}^{N}$, Math. Nachr., 278(2005), 1-10.

[12] P. H. RABINOWITZ, On a class of nonlinear Schrödinger equations, $Z$. Angew. Math. Phys., 43 (1992), 270-291. 
[13] B. RICCERI, A general multiplicity theorem for certain nonlinear equations in Hilbert spaces, Proc. Amer. Math. Soc., 133 (2005), 3255-3261.

[14] B. RICCERI, On a three critical points theorem, Arch. Math. (Basel), 75 (2002), 220-226.

[15] B. RICCERI, Existence of three solutions for a class of elliptic eigenvalue problems, Math. Comput. Modelling, 32 (2000), 1485-1494.

[16] W. M. NI and J. SERRIN, Nonexistence theorems for quasilinear partial differential equations, Rend. Circ. Mat. Palermo 8 (1985), 171-185.

[17] W. A. STRAUSS, Existence of solitary waves in higher dimensions, Comm. Math. Phys., 55 (1977), 149-162.

[18] I. G. TSAR'KOV, Nonunique solvability of certain differential equations and their connection with geometric approximation theory, Math. Notes, 75 (2004), 259-271.

[19] M. WILlEM, Minimax Theorems, Birkhäuser, Boston, 1995.

[20] E. ZEIDLER, Nonlinear Functional Analysis and its Applications, vol. III, Springer-Verlag, 1984.

Received 21 December 2005; accepted 2 December 2006

Published Online First 21 September 2007

To access this journal online: http://www.birkhauser.ch 